Article

\title{
Asymmetric Synthesis of Optically Pure Aliphatic Amines with an Engineered Robust $\omega$-Transaminase
}

\author{
Linhan Dong ${ }^{1,2}$, Qinglong Meng ${ }^{1}$, Carlos Ramírez-Palacios ${ }^{1,3}{ }^{\circledR}$, Hein J. Wijma ${ }^{1}{ }^{(D}$, \\ Siewert J. Marrink ${ }^{3}$ (D) and Dick B. Janssen ${ }^{1, *}$ \\ 1 Biotransformation and Biocatalysis Group, Groningen Biomolecular Sciences and Biotechnology \\ Institute (GBB), University of Groningen, Nijenborgh 4, 9747 AG Groningen, The Netherlands; \\ donglinhan1998@gmail.com (L.D.); q.meng@rug.nl (Q.M.); j.c.ramirez.palacios@rug.nl (C.R.-P.); \\ h.j.wijma@rug.nl (H.J.W.) \\ 2 College of Chemistry, Jilin University, 2699 Qianjin Avenue, Changchun 130012, China \\ 3 Molecular Dynamics Group, Groningen Biomolecular Sciences and Biotechnology Institute (GBB), \\ University of Groningen, Nijenborgh 7, 9747 AG Groningen, The Netherlands; s.j.marrink@rug.nl \\ * Correspondence: d.b.janssen@rug.nl; Tel.: +31-503-634-008
}

Received: 12 October 2020; Accepted: 8 November 2020; Published: 12 November 2020

\begin{abstract}
The production of chiral amines by transaminase-catalyzed amination of ketones is an important application of biocatalysis in synthetic chemistry. It requires transaminases that show high enantioselectivity in asymmetric conversion of the ketone precursors. A robust derivative of $\omega$-transaminase from Pseudomonas jessenii (PjTA-R6) that naturally acts on aliphatic substrates was constructed previously by our group. Here, we explore the catalytic potential of this thermostable enzyme for the synthesis of optically pure aliphatic amines and compare it to the well-studied transaminases from Vibrio fluvialis (VfTA) and Chromobacterium violaceum (CvTA). The product yields indicated improved performance of PjTA-R6 over the other transaminases, and in most cases, the optical purity of the produced amine was above $99 \%$ enantiomeric excess (e.e.). Structural analysis revealed that the substrate binding poses were influenced and restricted by the switching arginine and that this accounted for differences in substrate specificities. Rosetta docking calculations with external aldimine structures showed a correlation between docking scores and synthetic yields. The results show that PjTA-R6 is a promising biocatalyst for the asymmetric synthesis of aliphatic amines with a product spectrum that can be explained by its structural features.
\end{abstract}

Keywords: transamination; asymmetric synthesis; alkanamines; transaminase; computational modeling

\section{Introduction}

Optically pure amines are of great interest for the pharmaceutical and wider fine chemical industries as they can serve as intermediates in the preparation of a diversity of biologically active compounds [1-3]. For example, chiral amines are used as building blocks in the synthesis of pharmaceuticals used in the treatment of Alzheimer's disease [4] and malaria [5] or as antitumor agents [6]. The enzymatic production of such amines is widely studied for furthering the application of biocatalysis in environmentally benign synthetic processes $[7,8]$.

Transaminases (TAs) catalyze the transfer of amino groups from amines (amino donors) to aldehydes or ketones (amino acceptors), with pyridoxal-5' phosphate (PLP) as the cofactor (Scheme 1) [2,9]. Whereas $\alpha$-transaminases act on substrates bearing a carboxylate group flanking the carbon carrying the amine functionality that is exchanged, $\omega$-transaminases ( $\omega$-TAs) act on nonproteinogenic amino acids with the amine and carboxylate groups at terminal positions. Many $\omega$-TAs and related enzymes are called amine transaminases (ATAs) as they act on substrates that lack a 
carboxylate functionality. This relaxed substrate specificity allows amination of various ketones when added as amino acceptor [10]. Mechanistically, in the first half reaction, the amino donor aminates the PLP to form a pyridoxamine intermediate (PMP) in several proton transfer steps. In the second half reaction, the ketone acceptor binds to the PMP enzyme to form the aminated product and regenerate PLP (Scheme 1). Recent efforts to tailor the $\omega$-TA substrate scope have focused on stabilization of the Michaelis complex of the ketone substrate [11-14] with little or no attention to reaction intermediates. The external aldimine is a key intermediate in the transamination reaction. Depending on the half reaction under consideration, the external aldimine can either undergo proton abstraction by the catalytic lysine to form a quinonoid intermediate or abstract a proton from lysine to form a geminal diamine intermediate [15]. Both formation and conversion of the external aldimine go through the formation of high-energy transition state structures, and their corresponding reactions are rate-limiting. Therefore, we hypothesized that substrates capable of forming more stable external aldimine complexes would overall have better reactivities.

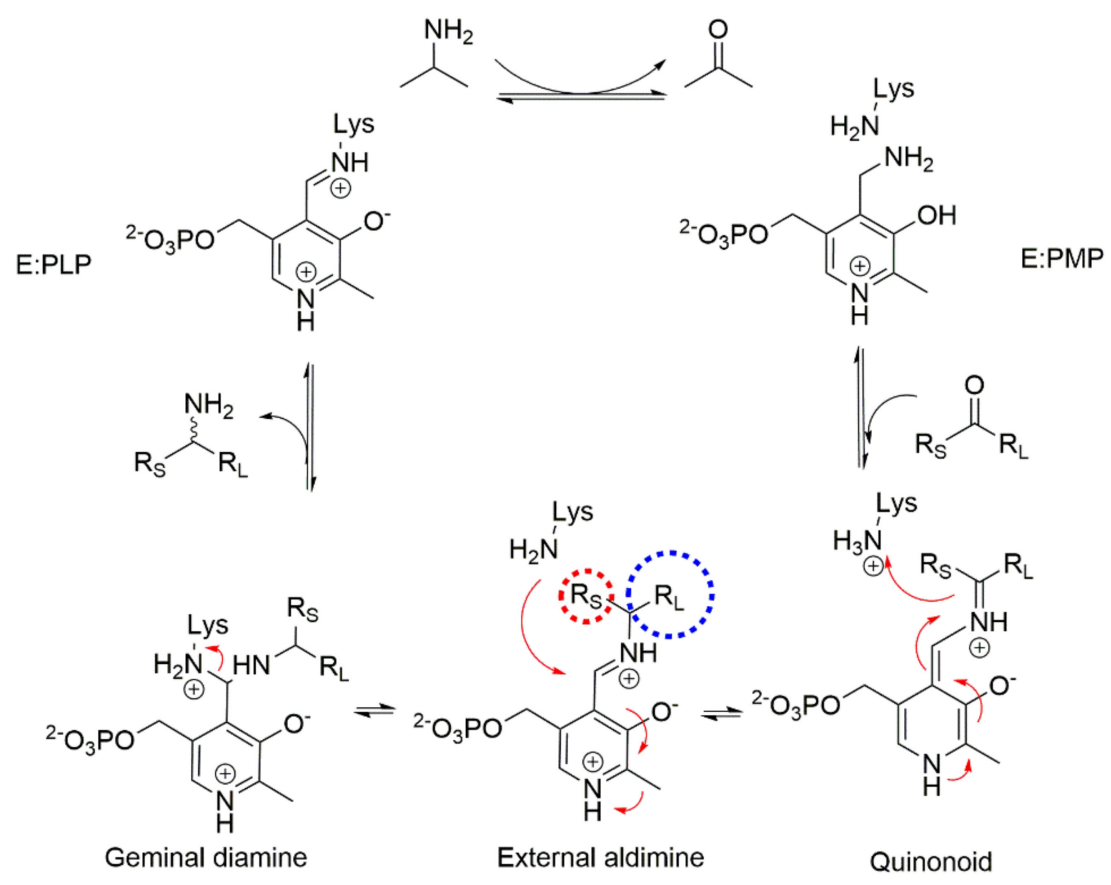

Scheme 1. $\omega$-Transaminase-catalyzed transamination with isopropylamine as the amino donor. The aldehyde/ketone substrate will bind to the pyridoxamine intermediate (PMP) enzyme to form an external aldimine. The relatively large $\left(R_{L}\right)$ or small $\left(R_{S}\right)$ ketone/amine rest groups are accommodated in the large or small binding pockets of the transaminase. The excess of isopropylamine drives the equilibrium toward the production of the target amine.

Prior work on the catalytic abilities of $\omega$-TAs indicates versatile substrate spectra and (S)- or $(R)$-enantioenrichment of amines formed by conversion of prochiral ketones [16]. Compared with traditional chemical synthesis, harsh conditions and heavy metal catalysts are avoided by enzymatic reactions, displaying the green aspects of biocatalysis [3,7]. Moreover, the use of cheap amine donors or regeneration of the amine donor in TA-catalyzed amination enables one-pot asymmetric synthesis [3,17]. Therefore, the production of optically pure amines applying $\omega$-TAs is considered promising from an application point of view. A large variety of transaminases that are active in the enzymatic synthesis of aromatic amines have been described [16]. In comparison, biocatalytic synthesis of chiral aliphatic amines is not well studied, and reports on asymmetric synthesis of aliphatic amines by TAs are scarce [16]. Nonetheless, aliphatic amines are frequently seen as important intermediates of pharmaceuticals and other biologically active compounds $[7,18]$. Thus, transaminases 
capable of producing various enantiopure aliphatic amines would diversify and strengthen the biocatalytic toolbox.

Apart from the need for an active enzyme for aliphatic amine synthesis, there are also several technical challenges concerning TA-catalyzed amine production. First, the reaction equilibrium needs to be shifted from the amino donor toward the production of the target amines. Often, this is achieved using an excess of the amino donor, sometimes accompanied by the removal of the side product $[7,16,19,20]$. Second, to use and fully dissolve a high concentration of the ketone substrate that should undergo amination, a cosolvent is sometimes added [1,21]. Besides overcoming equilibrium issues, the reaction rate may need to be accelerated using a high temperature. The availability of a robust $\omega$-TA is desired in such scenarios of high amino donor concentrations, presence of cosolvents, and high reaction temperatures [3].

The discovery [22] and characterization [23] of a class III (S)-selective $\omega$-TA from Pseudomonas jessenii (PjTA) were described previously. Identified as a key enzyme in the caprolactam degradation pathway of $P$. jessenii, PjTA converts 6-aminohexanoic acid (6-AHA) to 6-oxohexanoic acid (6-OHA). Our group has deepened the understanding of this enzyme by solving crystal structures and measuring its activities using over 40 different amine substrates [23]. A robust six-fold mutant of PjTA (PjTA-R6) was recently constructed using a framework for rapid enzyme stabilization by computational library design (FRESCO) [24]. The natural substrate of PjTA is a linear aliphatic $\omega$-amino acid, which suggests PjTA may have potential for the synthesis of aliphatic amines. Moreover, the robust mutant PjTA-R6 is expected to tolerate a high concentration of amino donor, the presence of cosolvent, and an enhanced reaction temperature [24]. Thus, in the work reported here, we explored $P j$ TA-R6 for the synthesis of aliphatic amines for which the wild-type PjTA was not considered as attractive due to its lower stability under harsh conditions. Considering their structural diversity and similarity to pharmaceutical building blocks, 10 aliphatic amines were selected for enzymatic production $[8,18]$. This included two amines with cycloalkyl substituents (C1 and $\mathbf{C} 2$ in Table 1$)$.

Table 1. Analytical yields and enantiomeric excesses for the production of A1-A5, B1-B3, and C1-C2 using a six-fold mutant of transaminase from Pseudomonas jessenii (PjTA-R6), a transaminase from Chromobacterium violaceum (CvTA), and a transaminase from Vibrio fluvialis (VfTA). ${ }^{1}$

\begin{tabular}{|c|c|c|c|c|c|c|c|}
\hline \multirow{2}{*}{$\begin{array}{c}\text { Amine } \\
\text { Target Code }\end{array}$} & \multirow{2}{*}{ Amine Structure } & \multicolumn{2}{|c|}{ PjTA-R6 } & \multicolumn{2}{|c|}{ CvTA } & \multicolumn{2}{|c|}{ VfTA } \\
\hline & & Yield ${ }^{1}(\%)$ & e.e. ${ }^{2}(\%)$ & Yield (\%) & e.e. $(\%)$ & Yield (\%) & e.e. $(\%)$ \\
\hline A1 & & 70 & 94 & 40 & 86 & 5 & n.d. ${ }^{3}$ \\
\hline A2 & & 80 & 98 & 34 & 91 & 12 & 41 \\
\hline A3 & & 51 & $>99$ & 23 & 91 & 14 & 88 \\
\hline A4 & & 51 & $>99$ & 44 & 90 & 41 & 92 \\
\hline A5 & & 42 & $>99$ & 46 & 88 & 35 & 94 \\
\hline B1 & & 95 & $>99$ & 3 & n.d. & 1 & n.d. \\
\hline
\end{tabular}


Table 1. Cont.

\begin{tabular}{|c|c|c|c|c|c|c|c|}
\hline \multirow{2}{*}{$\begin{array}{c}\text { Amine } \\
\text { Target Code }\end{array}$} & \multirow{2}{*}{ Amine Structure } & \multicolumn{2}{|c|}{ PjTA-R6 } & \multicolumn{2}{|c|}{ CvTA } & \multicolumn{2}{|c|}{$V f \mathrm{TA}$} \\
\hline & & Yield ${ }^{1}(\%)$ & e.e. ${ }^{2}(\%)$ & Yield (\%) & e.e. $(\%)$ & Yield (\%) & e.e. $(\%)$ \\
\hline B2 & & 81 & $>99$ & 3 & n.d. & 2 & n.d. \\
\hline B3 & & 48 & $>99$ & $<1$ & n.d. & 2 & n.d. \\
\hline $\mathrm{C} 1$ & & 42 & $>99$ & 1 & n.d. & $<1$ & n.d. \\
\hline C2 & $\underset{N}{\mathrm{NH}_{2}}$ & 63 & $>99$ & $<1$ & n.d. & 1 & n.d. \\
\hline
\end{tabular}

Reaction conditions: $1 \mathrm{~mL}$ volume, $20 \mathrm{mM}$ ketone substrate, 1 M isopropylamine, $0.5 \mathrm{mM}$ pyridoxal- $5^{\prime}$ phosphate (PLP), $1 \mathrm{mg} / \mathrm{mL}$ purified transaminase, $2 \%$ dimethyl sulfoxide (DMSO) (20\% for A5), $20 \mathrm{~h}, 56^{\circ} \mathrm{C}$ for $P j \mathrm{TA}-\mathrm{R} 6$ and $37^{\circ} \mathrm{C}$ for CvTA and VfTA. ${ }^{1}$ The yields obtained with PjTA-R6 are the averages of duplicates that differed less than $10 \% .{ }^{2}$ e.e.: enantiomeric excess. ${ }^{3}$ n.d.: not determined.enzymes. Noticeably, after $20 \mathrm{~h}$ of reaction, an enzyme precipitate was observed in the vials containing CvTA and VfTA but not in the reaction mixtures containing PjTA-R6. This agreed with our expectation that $P$ JTA-R6 would be more robust than the other two transaminases at high reaction temperature with excess amino donor present.

To compare the performance of PjAT-R6 to other well-characterized (S)-selective TAs, we included the enzymes from Chromobacterium violaceum (CvTA) and Vibrio fluvialis (VfTA). Through a systematic analysis of the substrate spectrum of various transaminases reported in different papers, Calvelage et al. [25] concluded that CvTA and VfTA have advantages in converting straight-chain amines over other transaminases. The relative performance of PjTA-R6 in comparison to CvTA and VfTA may help to evaluate the enzyme in terms of its significance within the enzymatic toolbox. Under the employed conditions, the yields of aliphatic amines obtained with PjTA-R6 were higher than with CvTA or VfTA, and the enantiopurity of the produced amines was also higher.

Aiming to understand the structural basis for the observed reactivities of PjTA-R6 and VfTA, we performed docking calculations of the external aldimine complexes of the tested substrates. For both enzymes, the interface energies of the external aldimine complexes correlated well with experimental reaction yields, indicating that biocatalytic scope can be accessed computationally for further redesign attempts. The docking complexes hinted to pivotal structural differences in the way PjTA-R6 and VfTA accommodate bulky or alkyl chains in the large substrate binding pocket.

\section{Results and Discussion}

\subsection{Asymmetric Synthesis of Aliphatic Amines}

To investigate the performance of $P j$ TA-R6 in the asymmetric synthesis of aliphatic amines and compare the results with CvTA and VfTA, amination reactions were conducted using the three enzymes and a series of keto substrates (Table 1). For these amination reactions, we adopted conditions with a 50 -fold excess of isopropylamine (IPA) as the amino donor (Scheme 1). This amino donor is relatively cheap [7] and highly water soluble, making its use in enzymatic aminations economically attractive. Moreover, IPA is transformed to acetone, whose volatility may simplify the work-up procedure [26]. The reaction temperature was $56^{\circ} \mathrm{C}$ for $P j \mathrm{TA}-\mathrm{R} 6$, given its high thermostability, and $37^{\circ} \mathrm{C}$ for $\mathrm{CvTA}$ and $V f T A$. Protein concentrations were the same for all three.

The asymmetric synthesis of aliphatic amines was successful as all ketone substrates were converted by PjTA-R6 and yielded predominantly $(S)$-amines with all yields exceeding $40 \%$ (Table 1 ). Whereas equilibrium may have been approached in some reactions (e.g., B1 with PjTA), the lower 
yields with most other substrates and especially in reactions catalyzed by CvTA and VfTA indicate that differences in yields were mainly determined by kinetics. In particular, amines A1 and $\mathbf{A} 2$ and branched amines B1 and $\mathbf{B} 2$ were well synthesized by PjTA-R6. Noticeably, using PjTA-R6, cyclic amine $\mathbf{C 2}$, carrying a cyclohexyl group substituent, was better synthesized than $\mathbf{C 1}$, which has a cyclopropyl group. This suggested that the general bulkiness of the larger substrate did not limit conversion. Chiral analysis of the amines formed by $P j$ TA-R6 confirmed the expected (S)-enantioenrichment, and all amines were produced at high enantiomeric excess (e.e.). The enantiopurities of the products indicated a dependence on the difference in size between the large $\left(\mathrm{R}_{\mathrm{L}}\right)$ and small $\left(\mathrm{R}_{\mathrm{S}}=-\mathrm{CH}_{3}\right)$ side substituents on the carbonyl carbon of the substrates. $\mathbf{A} \mathbf{1}\left(\mathrm{R}_{\mathrm{L}}=-\mathrm{CH}_{2} \mathrm{CH}_{3}\right)$ and $\mathbf{A} \mathbf{2}\left(\mathrm{R}_{\mathrm{L}}=-\mathrm{CH}_{2} \mathrm{CH}_{2} \mathrm{CH}_{3}\right)$ were produced with $94 \%$ and $98 \%$ e.e., respectively, while all the other amines were produced with $>99 \%$ e.e.

Under the employed conditions, the yields of straight-chain amines (A1-A5) in reactions using PjTA-R6 were generally better than those with CvTA and VfTA. For the production of A5, PjTA-R6 and CvTA displayed comparable results (Table 1). The yields found with PjTA-R6 were better in case of straight-chain aliphatic amines with shorter chains (A1-A2) than with amines with larger groups (A3-A5). In contrast, VfTA showed an improving trend in yields with an increasing chain length of the target amines. A previous study on the activity of $V f \mathrm{TA}$ toward a set of alkyl substrates also showed that the longer the side chain, the higher the activity [27]. For CvTA, no such trend was found. For the production of A1-A3, $P j$ TA-R6 generated higher yields by $28 \%$ or more than CvTA, while VfTA gave the lowest yields. The yields of A4 and $\mathbf{A} 5$ obtained with the three enzymes differed by less than $11 \%$. The general advantage of asymmetric synthesis using $P j$ TA-R6 was also clear from its better enantioselectivity. Whereas CvTA and VfTA produced linear aliphatic amines with e.e. $<94 \%$, all amines formed by PjAT-R6 had better than $94 \%$ e.e.

For amines with branched carbon chains (B1-B3) and cycloalkene groups (C1-C2), PjTA-R6 generated high product yields, similar to those of amines with linear substituents (Table 1). In particular, B1 was produced with $95 \%$ yield, which was the highest yield obtained with PjTA-R6. The advantage of PjTA-R6, compared with CvTA and VfTA, was again apparent from the higher yields. The conversion of amines with nonlinear substituents by $C v \mathrm{TA}$ and $V f \mathrm{TA}$ was not successful. This suggests that the presence of a nonlinear large rest group in the substrate prevents proper accommodation of the ketone or subsequent reaction intermediates in these enzymes. Overall, PjTA-R6 had a better performance in yield and excellent enantioselectivity, which makes $P j$ TA-R6 a promising candidate for the asymmetric amination of aliphatic ketones. The difference between the three enzymes can be due to kinetic and stability effects. Differences observed for the same enzyme should be attributed to kinetic differences, unless conversion is very high when equilibrium may be approached (Table 1, PjTA-R6 with A2 and B1). For the branched and cyclic substrates (B1-B3, C1, and C2), which were well converted by PjTA-R6 but poorly converted by CvTA and VfTA, low intrinsic catalytic activity of the latter two enzymes must be the cause of the observed differences.

\subsection{Role of Switching Arginine}

As mentioned, the native substrate of $P j \mathrm{TA}$ is the caprolactam biodegradation intermediate 6-aminohexanoic acid (6-AHA) [22]. The crystal structure of PjTA complexed with the external aldimine formed from this substrate unveils that the carboxylate group forms a salt bridge with a conserved arginine residue (Arg417) in the large pocket, probably contributing to substrate binding [23]. The arginine side chain switches toward a more outward position upon 6-AHA binding. Arg417 and most surrounding residues were kept during the thermostability engineering that yielded PjTA-R6 [24]. During the redesign of other class III (S)-selective transaminases, the switching arginine is mostly preserved as amino donors or acceptors often feature a carboxylate functionality [27-29]. However, in $V f T A$, Arg415 has been mutated to open up more space in the large pocket $[27,29,30]$. For the synthesis of aliphatic alkylamines with IPA as amino donor, neither Arg415 nor Arg417 of VfTA and PjAT-R6, respectively, are required, and the iminium group can even produce repulsive interactions. 
To examine whether Arg417 still has a role in substrate binding when using isopropylamine as amino donor and an amino acceptor substrate lacking a carboxylate functionality, the R417L mutant of PjTA-R6 was prepared and tested in the same reaction scheme as for PjTA-R6. Thermal shift fluorescence assays showed that the thermostability of the R6-R417L enzyme was similar to that of the parent $P j$ TA-R6 $\left(\Delta T_{\mathrm{m}}{ }^{\text {app }}<1{ }^{\circ} \mathrm{C}\right)$. The yields of the enzymatic amination using the mutant $\mathrm{R} 417 \mathrm{~L}$ remained at the same level as that of PjTA-R6. Yields of A1 and A2 obtained with R6-R417L (60\% and $69 \%$, respectively) were only slightly lower than that with $P j \mathrm{TA}-\mathrm{R} 6$ (70\% and 80\%). As for A3-A5, the yields obtained with R6-R417L (54\%, 50\%, and 47\%) were very close to the yields with PjTA-R6. Apparently, the positive charge of the arginine did not hinder activity with the apolar aliphatic acceptors tested here. A possible explanation is conformational flexibility of the aliphatic group of the substrates or of the Arg417, which may avoid close contact with the positively charged iminium function, unlike what is observed in the 6-AHA-bound PjTA structure [23]. We anticipate that Arg417 can be replaced for future engineering needs, e.g., aimed at expanding the binding site for more bulky substrates.

\subsection{Structure-Activity Relationship Obtained by Docking}

Accommodation of the external aldimine docking complexes formed with different substrates by PjTA-R6 and VfTA were examined to find a structural explanation for the observed activity profiles (Table 1). The substrate-binding pockets of these TAs were positioned at the dimeric interface and consisted of residues from both subunits. Because the small substituent of the tested molecules was always a methyl group, the structure of the binding pocket that accommodates the large substituent was crucial for differences in substrate specificity. The large binding pocket accommodated the carboxyalkyl moiety or large substituent of 6-AHA in PjTA and the phenyl substituent of (S)-1-phenylethylamine in VfTA. Structural alignment of PjTA-R6 with CvTA (40\% sequence identity, RMSD $1.28 \AA$ ) and with $V f T A$ ( $41 \%$ sequence identity, RMSD $1.20 \AA$ ) indicated a high overall similarity, although with local differences in the large binding pockets (Figure 1). The side chains of Leu57, Tyr151, Arg417, Met419, Ala230, Trp58, Val260, and Ile261 formed a largely nonpolar binding pocket in PjTA-R6. The corresponding pockets of CvTA and VfTA were also hydrophobic. To examine the features that potentially led to variations in substrate specificities and conferred PjTA-R6 with high reactivity toward a variety of bulky amines, structures of enzyme complexed with product-derived external aldimines were generated by Rosetta docking.

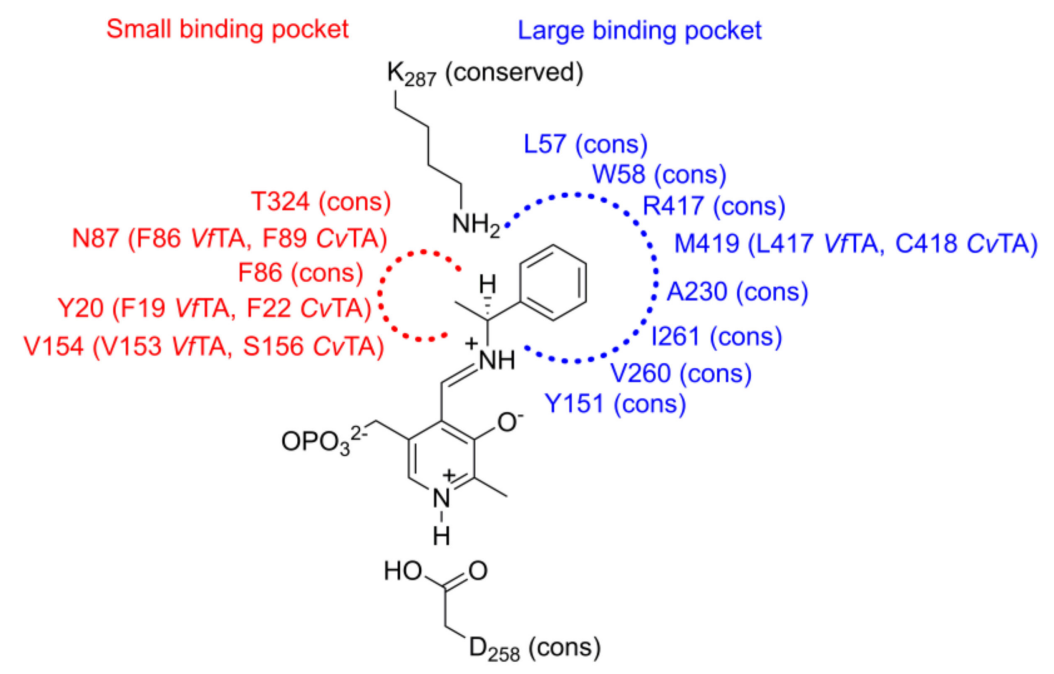

Figure 1. Schematic representation of the binding pocket of PjTA-R6 bearing the external aldimine intermediate (PLP Schiff base with (S)-1-phenylethylamine) and comparison of surrounding residues to CvTA and VfTA. The residues in the large binding pocket are in blue. The residues colored red are in the small binding pocket. 
The Rosetta interface energies obtained from docking the external aldimine complexes of the 10 different substrates showed a clear correlation with the experimental data observed for both VfTA and PjTA-R6 (Figure 2). Nevertheless, outliers were present in both plots. The most prominent outlier in the VfTA dataset was compound B3, which exhibited a higher docking score than would be expected based on the experimental yield. B3 was also one of the three outliers in PjTA-R6, along with A4 and A5. The latter two were the only two compounds in the PjTA-R6 dataset where the switching arginine Arg417 in the docking complex was kept in a crystal-like "out" conformation (discussed later), whereas all the other compounds (A1-A3, B1-B3, and C1-C2) showed Arg417 in an "in" conformation. The lower than expected yield for A5 with PjTA-R6 might also be due to the higher concentration of dimethyl sulfoxide (DMSO) used to dissolve this substrate. The correlation between interface energies and experimental yields suggested that structural differences in the binding pockets could have accounted for most of the observed differences in activity within a set of substrates tested with the same enzyme. Because at least one substrate for each enzyme was very well converted, low yields for several substrates, such as B1-C2 for CvTA and VfTA, must be due to kinetic causes rather than rapid enzyme inactivation. However, differences in obtained yields between different enzymes might be related to both kinetic and stability differences under the reaction conditions.
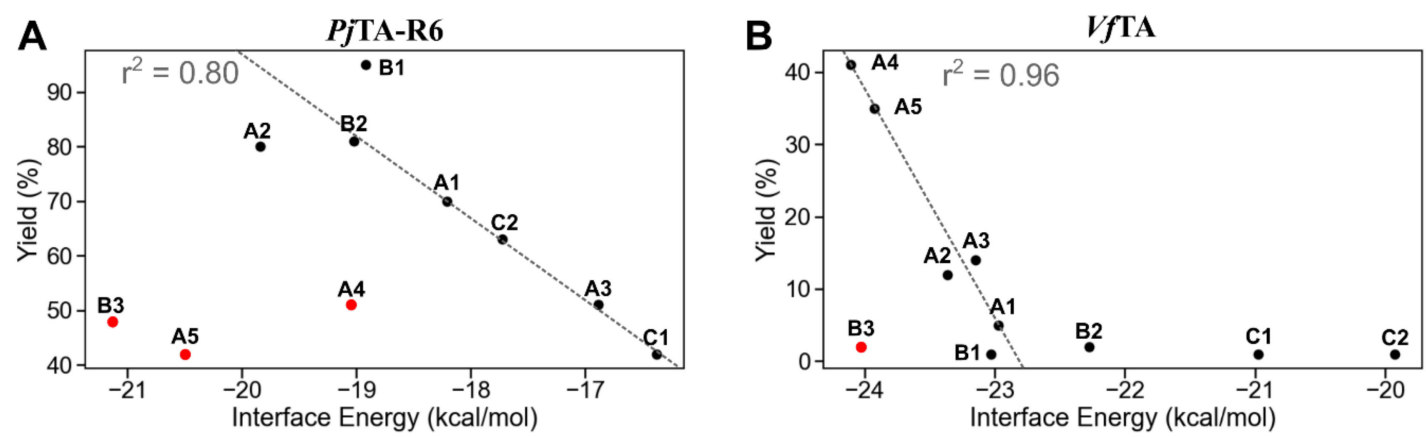

Figure 2. Interface energies of external aldimine complexes show a correlation with the observed activities. (A) PjTA-R6 and (B) VfTA. Outliers (red dots) were not considered for the linear regression (dotted line). For PjTA-R6, this means the regression was made using compounds A1, A2, A3, B1, B2, $\mathrm{C} 1$, and $\mathrm{C} 2$. In the case of $V f \mathrm{TA}, \mathrm{B} 1, \mathrm{~B} 2, \mathrm{C} 1$, and $\mathrm{C} 2$ were not considered for the linear regression as the product was hardly detectable $(<2 \%)$. The Rosetta interface energy correctly predicted that $\mathrm{B} 2, \mathrm{C} 1$, and C2 would have very low yields; hence, these compounds were not outliers.

The activity data suggested that PjTA-R6 could accommodate bulkier substituents than VfTA (Table 1). The docking simulations revealed that the conformational freedom of the switching arginine played a large role in the ability of PjTA-R6 to accommodate bulky substrates. In both VfTA and PjTA-R6, the switching arginine (Arg415 and Arg417, respectively) could adopt one of two conformations that allowed the binding of hydrophobic substituents in the large pocket (Figure 3). We labeled these Arg conformations as "in" and "out" to indicate whether the guanidium group was pointing inward (toward) the PLP or outward. The "in" and "out" arginine conformations in $V f \mathrm{TA}$ and PjTA-R6, respectively, were crystallized. In the known VfTA structures (PDB codes: 4E3Q, 4E3R, 5ZTX, and 3NUI), Arg415 was stabilized in the inward conformation by a hydrogen bond with Trp57. As such, the "in" conformation appeared to be the most stable for the PLP-bound enzyme and the apo-enzyme. On the other hand, the "out" arginine conformation prevailed in the known PjTA structures (PDB codes: 6TB1, 6TB0, 6G4B, 6G4C, 6G4D, 6G4E, and 6G4F), where Arg 417 was stabilized by electrostatic interactions, either with Ser87 in the wild-type PjTA or with Asn87 in PjTA-R6. In PjTA-R6, Ser87 has been mutated to Asn to improve the enzyme's thermostability $[15,16]$. The natural substrate of PjTA is 6-AHA, where the role of the switching arginine is to form a salt bridge with the carboxyl group of the substrate (code: 6G4E). PjTA-R6 and VfTA exhibited structurally different "in" and "out" Arg conformations. The outward conformation in VfTA placed the Arg415 side chain 
in a sandwich-like configuration between Trp57 and Asn53, reducing the ability of Trp57 to freely rotate in response to the presence of a bulky substrate. On the other hand, the "out" conformation of PjTA-R6 moved Arg417 away from the binding pocket, locking Arg417 with Asn87 via a polar interaction, thereby both opening up more space in the large pocket and allowing free rotation of Trp58. The inward conformations of Arg415/Arg417 in VfTA and PjTA-R6 could also account for the difference in reactivity toward bulky substrates. In the "in" conformation, the switching arginine of PjTA-R6 made hydrogen bonding contacts with the backbone of Ala230, leaving enough space in the large pocket for bulky substituents. In contrast, VfTA locked Arg415 with the indole amino group of Trp57 via hydrogen-bonding interactions, offering less space in the large pocket. Mutations of Trp57 have been carried out, yielding good reactivities toward both alkyl and aromatic substrates [20]. Arg415 from $V f$ TA could not reach the backbone of the equivalent Ala228 due to the presence of Leu417, which corresponded to Met419 in PjTA-R6. These differences in both the "in" and "out" conformations can explain the distinct catalytic performance of the two enzymes toward bulky substrates.

A

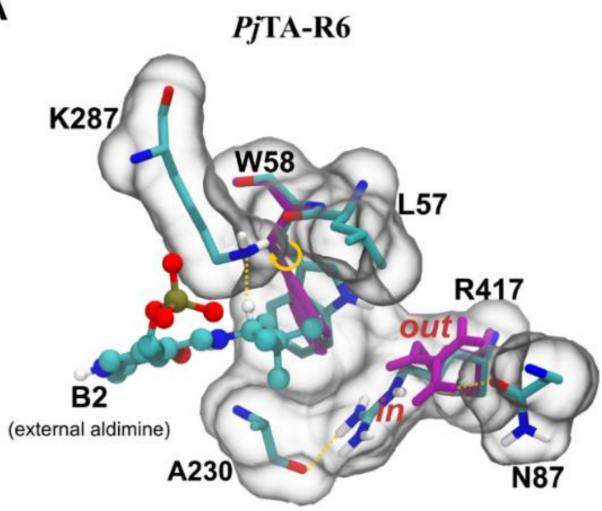

B

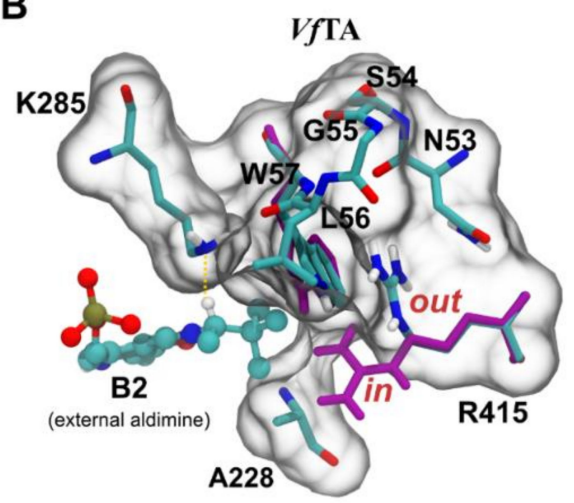

Figure 3. Docked external aldimine structures of 3,3-dimethyl-2-butylamine B2, representative of a products with bulky substituents. (A) In PjTA-R6, the tert-butyl substituent can be easily accommodated in the large pocket by a $40^{\circ}$ rotation of Trp58 (yellow arrow). Arg417 does not induce protein-ligand clashes in either the "in" or the "out" orientation. (B) In VfTA, both the "in" and the "out" conformations of Arg415 prevent a rotation of Trp57 necessary to accommodate the tert-butyl substituent. In both panels, protein structures are shown in licorice representation, and the crystal structure conformations of residues Arg417/Arg415 and Trp58/Trp57 are colored magenta (PDBs 4Q3H for VfTA and 6TB1 for PjTA-R6). The external aldimine structure of $\mathbf{B} 2$ is shown in CPK representation. Figures were rendered using VMD (Visual Molecular Dynamics).

An additional difference between PjTA-R6 and VfTA was the effect of increasing the chain length of the alkyl substituent on activity (i.e., substrates A1-A5). The experimental data showed that longer alkyl substituents negatively impacted conversion by PjTA-R6 (Table 1). On the contrary, VfTA (and CvTA) showed better activities with longer alkyl substituents, as also found by Cho et al. in a study aimed at redesigning the substrate specificity of $V f T A$ [27]. Rosetta-generated docking models of the external aldimine intermediates of A1-A5 indicated that $V f \mathrm{TA}$ accommodated the increasingly longer alkyl chain in the large pocket by pointing the terminal methyl group of the alkyl chain toward the entry tunnel (Figure 4B). In the case of $P j$ TA-R6, the equivalent space was partially blocked by Arg417 in both the "in" and "out" conformations. The terminal methyl group of the alkyl chain of A4 and A5 was still perfectly able to fit in the large pocket of $P j$ TA-R6, albeit less favorably (Figure 4A) than compounds with shorter alkyl substituents (i.e., A1-A3). The negative effect of Arg417 on the activity of PjTA-R6 was minor, as demonstrated by the fact that mutation R417L offered only a marginal improvement in the yields of amines A4 and A5. Furthermore, the large pocket of PjTA-R6 contained more polar residues than $V f T A$, thereby offering a less suitable environment to accommodate the lengthening alkyl chain of the A1-A5 series. All in all, while the catalytic activity of PjTA-R6 decreased for long alkyl 
chains, PjTA-R6 still gave higher yields and higher enantioselectivities than $V f$ TA, making $P j \mathrm{TA}-\mathrm{R} 6$ valuable for synthesis of enantiopure alkyl amines.

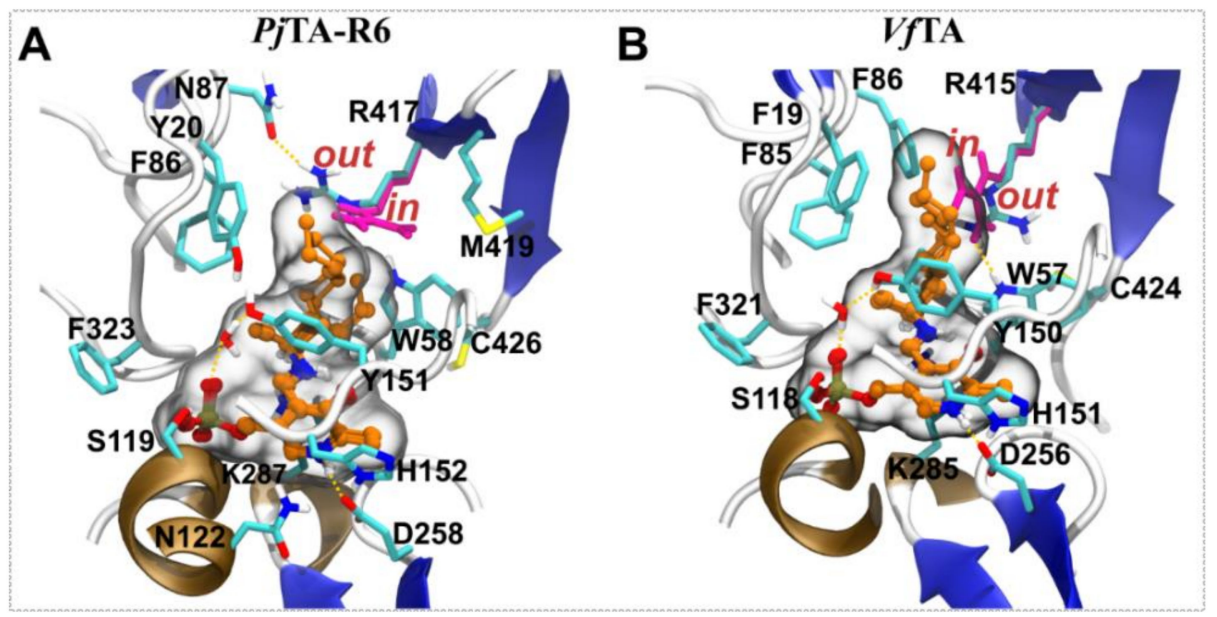

Figure 4. Docking structures of the external aldimine intermediates of amines A1-A5. (A). PjTA-R6 and (B) VfTA. The "out" conformation of the switching arginine is preferred for longer substituents (A4 and A5), whereas the shorter alkyl substituents (A1 to A3) show no preference for either conformation. The large binding site of PjTA-R6 is less hydrophobic than the binding site of $V f T A$ (N87, Y20, and F86 for PjTA-R6 and F86, F19, and F85 for VfTA, respectively). The superimposed external aldimine structures of A1-A5 are shown in CPK representation. The "in" conformation of Arg417/Arg415 is colored magenta.

\section{Materials and Methods}

\subsection{Materials}

Isopropylamine, amines (A1-A5, B1-B3, and C1-C2), and the corresponding ketones were purchased from Sigma-Aldrich (St. Louis, MO, USA) or Acros Organics (Fair Lawn, NJ, USA). PLP was purchased from Fisher Scientific (Pittsburgh, PA, USA). Other chemicals were from the same suppliers or Merck Millipore (Burlington, MA, USA).

\subsection{Enzyme Expression and Purification}

The design, isolation, and properties of the PjTA-R6 mutant have been previously described [24]. The PjTA-R6-R417L mutant was created by QuikChange site-directed mutagenesis. Primers were designed by the QuikChange Primer Design Program of Agilent Technologies (Santa Clara, CA, USA). The mutations were verified by DNA sequencing (Eurofins Genomics, Ebersberg, Germany). After sequencing, the mutated plasmids were transformed into E. coli BL21(DE3) for expression. The expression and purification of PjTA-R6, PjTA-R6-R417L, CvTA, and VfTA were performed as previously described. Specific activities of purified enzymes in the deamination of (S)-1-phenylethylamine were $47 \mathrm{U} / \mathrm{mg}$ (at $70{ }^{\circ} \mathrm{C}$, spectrophotometric acetophenone production assay [24]) or 1.6 and $1.0 \mathrm{U} / \mathrm{mg}$ (at $30^{\circ} \mathrm{C}$, coupled enzyme assay using pyruvate as amino acceptor [23]) for $P j \mathrm{TA}, \mathrm{Cv} \mathrm{TA}$, and $V f \mathrm{TA}$, respectively. 


\subsection{Thermal Shift Assay}

Apparent melting temperatures $\left(T_{\mathrm{m}}{ }^{\text {app }}\right)$ were determined by fluorescence-based thermofluor assay [31]. Specifically, samples of $5 \mu \mathrm{L} 50$-fold diluted SYPRO orange and $20 \mu \mathrm{L} 0.5 \mathrm{mg} / \mathrm{mL}$ enzyme solution were added to an IQ 96-well PCR plate (Bio-Rad, Hercules, CA, USA) and mixed thoroughly in the wells. The plates were sealed with Microseal B adhesive sealer (Bio-Rad, USA) and heated from 20 to $99{ }^{\circ} \mathrm{C}$ in a MyiQ real-time PCR machine (Bio-Rad, USA) with a linear gradient of increasing temperature $\left(1{ }^{\circ} \mathrm{C} / \mathrm{min}\right)$. The temperature at the maximum rate of fluorescence change $(\mathrm{dRFU} / \mathrm{dT})$ was taken as $T_{\mathrm{m}}$ app [32].

\subsection{Amination Reactions and Product Derivatization}

Enzyme reactions were carried out in a $1 \mathrm{~mL}$ reaction system containing $50 \mathrm{mM}$ potassium phosphate, $\mathrm{pH}$ 8.0, with $20 \mathrm{mM}$ ketone substrate, $1 \mathrm{M}$ isopropylamine, $0.5 \mathrm{mM} \mathrm{PLP}, 1 \mathrm{mg} / \mathrm{mL}$ enzyme, and $2 \%$ DMSO (20\% for A5). Reactions were started by addition of substrate and carried out at $56{ }^{\circ} \mathrm{C}$ for PjTA-R6 and R417L and $37^{\circ} \mathrm{C}$ for CvTA and VfTA over $20 \mathrm{~h}$. Reactions were stopped by adding $200 \mu \mathrm{L} 10 \mathrm{M} \mathrm{NaOH}$, after which $100 \mu \mathrm{L}$ samples were taken from the basified solutions and extracted once with $300 \mu \mathrm{L}$ ethyl acetate.

For derivatization, the organic layer was supplied with $300 \mu \mathrm{L}$ acetic anhydride and 4-dimethylaminopyridine (DMAP) [1], and the solution was shaken at room temperature and $450 \mathrm{rpm}$ for $1 \mathrm{~h}$. Following washing with $500 \mu \mathrm{L}$ water, the organic layer containing the derivatized product was collected and dried over magnesium sulfate.

\subsection{Quantification and Optical Purity}

The derivatized products were analyzed by gas chromatography with flame ionization detection on a heptakis-(2,6-di-O-methyl-3-O-pentyl)- $\beta$-cyclodextrin column using $\mathrm{He}$ at a flow rate of $1 \mathrm{~mL} / \mathrm{min}$. The following settings were applied to the analysis of all products and their corresponding commercial references: temperature program: $80^{\circ} \mathrm{C}$ for $6.5 \mathrm{~min}, 10^{\circ} \mathrm{C} / \mathrm{min}$ to $160^{\circ} \mathrm{C}$, and $20^{\circ} \mathrm{C} / \mathrm{min}$ to $200^{\circ} \mathrm{C}$ for $10 \mathrm{~min}$; total run time: $26.5 \mathrm{~min}$; inlet temperature: $220^{\circ} \mathrm{C}$; and injection volume: $1 \mu \mathrm{L}$. To quantify the product, the peaks of the enantiomers were summed up and compared with a commercial amine reference (see Supplementary Materials). To analyze the optical purity of the products, the peaks of different enantiomers were mostly identified with commercial chiral amine references (see Supplementary Materials). The e.e. values were calculated from the peak areas of the (S)- and (R)-amines.

\subsection{Computational Modeling}

The coordinates for the (S)-amino products of the tested compounds (A1-A5, B1-B3, and C1-C2) were generated in Avogadro (Hanwell et al., 2012; https://avogadro.cc/) [26] from their respective SMILES (Simplified Molecular Input Line Entry System) string and optimized by 500 steps of steepest descent energy minimization in the MMFF94 force field. The initial coordinates for the external aldimine forms of the tested substrates were created by superimposing the amino group of the abovementioned (S)-amino products to the amino group of the PLP cofactor, whose coordinates were obtained from the crystal structure (PDB: 4E3Q). After superimposition, one of the nitrogen atoms was deleted (the cofactor's), and a covalent bond was added between $\mathrm{C}^{\prime}$ ' and the remaining nitrogen atom (the amino substrate's) to form the corresponding Schiff base. A rotamer library for each ligand was generated in YASARA [33]. For each external aldimine structure, 1000 rotamers were generated by random perturbation of the heavy-atom dihedrals (SampleDih YASARA routine) while maintaining the cofactor atoms frozen. Pairwise RMSD (root-mean-square deviation) between all rotamers within each library was used for selecting unique rotamers (cutoff $<0.05 \AA$ ), resulting in 200-300 unique rotamers for each ligand. 
Structure coordinates for PjTA-R6 [24] and VfTA [30] were obtained from the PDB databank (6TB1 and $4 \mathrm{E} 3 \mathrm{Q}$, respectively). The crystal structures were prepared for docking by first implementing the CleanObj YASARA routine, which added explicit hydrogens to the structure, and converting Se-Met residues into canonical Met. Protonation states of residues Asp, Glu, His, and Lys were calculated by an automatic YASARA routine at $\mathrm{pH} 7$ [33]. The resulting scaffold structure was stripped of any nonprotein residues.

The scaffold protein structure was relaxed by the Rosetta (v2020.11) Relax [34] algorithm with the following command-line arguments: -ignore_unrecognized_res, -relax:constrain_relax_to_start_coords, -relax:coord_constrain_sidechains, -relax:ramp_constraints false, -use_input_sc, -correct, -no_his_his_pairE, -no_optH false, -flip_HNQ. Binding poses of external aldimine complexes were generated using the Rosetta Enzyme Design application [35]. One randomly selected rotamer from each conformer library was overlapped to the binding site (subunit A) of PjTA-R6 or VfTA by RMSD alignment to the cofactor heavy atoms. The resulting structure served as input for the docking calculations. During the docking simulations, residues with $\mathrm{C} \alpha$ closer than $10 \AA$ from any ligand heavy atom were allowed to repack. For PjTA-R6, residues 20, 87, 59, 118, 119, 151, 152, 225, 259, 287, 292, and 324 were set to NATRO (natural rotamer, kept fixed). For VfTA, residues 19, 53, 115, $118,121,150,151,256,258$, and 322 were set to NATRO. No constraints were used in the production runs. Additionally, the following command-line arguments were used for the Rosetta docking: -enzdes, -cst_predock, -cst_design, -cut1 0.0 -cut2 0.0, -cut3 8.0, -cut4 10.0, -cst_min, -chi_min, -bb_min, -packing::use_input_sc, -packing::soft_rep_design, -design_min_cycles 3, -ex1:level 4, -ex1:level 4, -ex1aro:level 4, -ex2aro:level 4 . A total of 200 docking complexes were generated for each ligand, and the average interface energies were computed and compared against the experimental yield. Figures were generated using VMD (http://www.ks.uiuc.edu/Research/vmd/).

\section{Conclusions}

PjTA-R6 was capable of converting various aliphatic ketones into the corresponding amines with good activity, including when compared to $C v \mathrm{TA}$ and $V f \mathrm{TA}$. The scope of aliphatic amines that can be asymmetrically synthesized spans from amines with linear (A1-A5) or branched substituents (B1-B3) to lesser studied amines with a cycloalkyl moiety (C1-C2). The enantioselectivity of PjTA-R6 was ideal for most cases, which plays in favor of using this enzyme for chiral amine production. PjTA-R6 catalyzed amination at a relatively high temperature $\left(56^{\circ} \mathrm{C}\right)$. Its robustness allows use of the enzyme under beneficial conditions for enzymatic amination, e.g., high temperature, higher amino donor concentration, and presence of cosolvent for better substrate and product solubility. The mutant R6-R417L showed a comparable performance to PjTA-R6, indicating that the switching arginine Arg417 is replaceable when a carboxylate functionality is absent from the keto substrate and the amino donor.

The interface energies obtained with Rosetta docking simulations of the external aldimine reaction intermediates showed a clear correlation with yield in amine synthesis, both in case of PjTA-R6 and VfTA. Further comparison of the docked structures unveiled differences in the conformations of the switching arginine that interacts with the native substrates of PjTA-R6 and VfTA. In VfTA, the conformational freedom of the switching arginine is more restricted than in $P j$ TA-R6 and does not allow $V f$ TA to accommodate bulky substrates. Overall, the binding site of PjTA-R6 is better at adapting to fit bulkier substituents than the related enzyme $V f \mathrm{TA}$. The observation that the relative reactivity of $P j$ TA-R6 can be rationalized by docking simulations and energy calculations suggests that these computational tools can support the design of variants with expanded or shifted substrate spectrum, which is important for furthering applicability in green chemistry.

Supplementary Materials: The following are available online at http://www.mdpi.com/2073-4344/10/11/1310/s1, Figure S1: The chromatograms of 20, 2, and $0.2 \mathrm{mM} \mathrm{A3}$ sample after derivatization; Figure S2: Representative chromatogram of PjTA-R6-catalyzed transamination of 2-butanone yielding sec-butylamine (A1) after product derivatization; Figure S3: Representative chromatogram of PjTA-R6-catalyzed transamination of 2-pentanone yielding 2-aminopentane (A2) after product derivatization; Figure S4: Representative chromatogram of PjTA-R6-catalyzed transamination of 2-hexanone yielding 2-aminohexane (A3) after product derivatization. 
Figure S5: Representative chromatogram of PjTA-R6-catalyzed transamination of 2-heptanone yielding 2-aminoheptane (A4) after product derivatization; Figure S6: Representative chromatogram of PjTA-R6-catalyzed transamination of 2-octanone yielding 2-aminooctane (A5) after product derivatization; Figure S7: Representative chromatogram of PjTA-R6-catalyzed transamination of 3-methyl-2-butanone yielding 3-methyl-2-butanamine (B1) after product derivatization; Figure S8: Representative chromatogram of PjTA-R6-catalyzed transamination of 3,3-dimethyl-2-butanone yielding 3,3-dimethyl-2-butanamine (B2) after product derivatization; Figure S9: Representative chromatogram of PjTA-R6-catalyzed transamination of 4-methyl-2-pentanone yielding 4-methyl-2-pentanamine (B3) after product derivatization; Figure S10: Representative chromatogram of PjTA-R6-catalyzed transamination of 1-cyclopropylethanone yielding 1-cyclopropylethanamine (C1) after product derivatization; Figure S11: Representative chromatogram of PjTA-R6-catalyzed transamination of 1-cyclohexylethanone yielding 1-cyclohexylethanamine (C2) after product derivatization; Figure S12: SDS-PAGE analysis of purified CvTA, VfTA, and PjTA-R6; Table S1: The retention times of the derivatized products.

Author Contributions: Conceptualization, Q.M., C.R.-P., H.J.W., S.J.M., and D.B.J.; formal analysis, L.D. and C.R.-P.; funding acquisition, Q.M., C.R.-P., L.D., S.J.M., and D.B.J.; investigation, L.D., C.R.-P., and Q.M.; methodology, L.D., Q.M., and C.R.-P.; supervision, H.J.W., S.J.M., and D.B.J.; validation, Q.M. and C.R.-P.; visualization, L.D., C.R.-P., and Q.M.; writing—original draft, L.D., Q.M., and C.R.-P.; writing-review and editing, H.J.W., S.J.M., and D.B.J. All authors have read and agreed to the published version of the manuscript.

Funding: The research of H.J.W. was supported by the Dutch Ministry of Economic Affairs through BE-Basic, grant FS02.005.

Acknowledgments: L.D. thanks the University of Groningen and Jilin University for the scholarships they provided. Q.M. thanks the China Scholarship Council for a PhD fellowship. C.R.P. thanks CONACYT for the doctoral fellowship.

Conflicts of Interest: The authors declare no conflict of interest.

\section{References}

1. Koszelewski, D.; Lavandera, I.; Clay, D.; Rozzell, D.; Kroutil, W. Asymmetric Synthesis of Optically Pure Pharmacologically Relevant Amines Employing w-Transaminases. Adv. Synth. Catal. 2008, 350, 2761-2766. [CrossRef]

2. Ghislieri, D.; Turner, N.J. Biocatalytic Approaches to the Synthesis of Enantiomerically Pure Chiral Amines. Top. Catal. 2014, 57, 284-300. [CrossRef]

3. Patil, M.D.; Grogan, G.; Bommarius, A.; Yun, H. Recent Advances in $\omega$-Transaminase-Mediated Biocatalysis for the Enantioselective Synthesis of Chiral Amines. Catalysts 2018, 8, 254. [CrossRef]

4. Rösler, M.; Anand, R.; Cicin-Sain, A.; Gauthier, S.; Agid, Y.; Dal-Bianco, P.; Stähelin, H.B.; Hartman, R. Efficacy and safety of rivastigmine in patients with Alzheimer's disease: International randomised controlled trial. BMJ 1999, 318, 8. [CrossRef] [PubMed]

5. Hoye, T.R.; Chen, M. Total synthesis of (ent)-korupensamine D. Tetrahedron Lett. 1996, 37, 3099-3100. [CrossRef]

6. Chen, X.; Chen, J.; De Paolis, M.; Zhu, J. Synthetic Studies toward Ecteinascidin 743. J. Org. Chem. 2005, 70, 4397-4408. [CrossRef]

7. Kelly, S.A.; Pohle, S.; Wharry, S.; Mix, S.; Allen, C.C.R.; Moody, T.S.; Gilmore, B.F. Application of $\omega$-Transaminases in the Pharmaceutical Industry. Chem. Rev. 2018, 118, 349-367. [CrossRef]

8. Simon, R.C.; Richter, N.; Busto, E.; Kroutil, W. Recent Developments of Cascade Reactions Involving $\omega$-Transaminases. ACS Catal. 2014, 4, 129-143. [CrossRef]

9. Eliot, A.C.; Kirsch, J.F. Pyridoxal Phosphate Enzymes: Mechanistic, Structural, and Evolutionary Considerations. Annu. Rev. Biochem. 2004, 73, 383-415. [CrossRef]

10. Slabu, I.; Galman, J.L.; Lloyd, R.C.; Turner, N.J. Discovery, Engineering, and Synthetic Application of Transaminase Biocatalysts. ACS Catal. 2017, 7, 8263-8284. [CrossRef]

11. Voss, M.; Das, D.; Genz, M.; Kumar, A.; Kulkarni, N.; Kustosz, J.; Kumar, P.; Bornscheuer, U.T.; Höhne, M. In Silico Based Engineering Approach to Improve Transaminases for the Conversion of Bulky Substrates. ACS Catal. 2018, 8, 11524-11533. [CrossRef]

12. Han, S.-W.; Kim, J.; Cho, H.-S.; Shin, J.-S. Active Site Engineering of $\omega$-Transaminase Guided by Docking Orientation Analysis and Virtual Activity Screening. ACS Catal. 2017, 7, 3752-3762. [CrossRef]

13. Sirin, S.; Kumar, R.; Martinez, C.; Karmilowicz, M.J.; Ghosh, P.; Abramov, Y.A.; Martin, V.; Sherman, W. A Computational Approach to Enzyme Design: Predicting w-Aminotransferase Catalytic Activity Using Docking and MM-GBSA Scoring. J. Chem. Inf. Model. 2014, 54, 2334-2346. [CrossRef] [PubMed] 
14. Dourado, D.F.A.R.; Pohle, S.; Carvalho, A.T.P.; Dheeman, D.S.; Caswell, J.M.; Skvortsov, T.; Miskelly, I.; Brown, R.T.; Quinn, D.J.; Allen, C.C.R.; et al. Rational Design of a (S)-Selective-Transaminase for Asymmetric Synthesis of (1S)-1-(1,1'-biphenyl-2-yl)ethanamine. ACS Catal. 2016, 6, 7749-7759. [CrossRef]

15. Cassimjee, K.E.; Manta, B.; Himo, F. A quantum chemical study of the $\omega$-transaminase reaction mechanism. Org. Biomol. Chem. 2015, 13, 8453-8464. [CrossRef]

16. Koszelewski, D.; Tauber, K.; Faber, K.; Kroutil, W. $\omega$-Transaminases for the synthesis of non-racemic $\alpha$-chiral primary amines. Trends Biotechnol. 2010, 28, 324-332. [CrossRef]

17. Park, E.-S.; Malik, M.S.; Dong, J.-Y.; Shin, J.-S. One-Pot Production of Enantiopure Alkylamines and Arylalkylamines of Opposite Chirality Catalyzed by $\omega$-Transaminase. ChemCatChem 2013, 5, 1734-1738. [CrossRef]

18. Liu, T.-L.; Wang, C.-J.; Zhang, X. Synthesis of Chiral Aliphatic Amines through Asymmetric Hydrogenation. Angew. Chem. Int. Ed. 2013, 52, 8416-8419. [CrossRef]

19. Shin, J.-S.; Kim, B.-G. Asymmetric Synthesis of Chiral Amines with w-Transaminase. Biotechnol. Bioeng. 1999, 65, 206-211. [CrossRef]

20. Fuchs, M.; Farnberger, J.E.; Kroutil, W. The Industrial Age of Biocatalytic Transamination: The Industrial Age of Biocatalytic Transamination. Eur. J. Org. Chem. 2015, 2015, 6965-6982. [CrossRef]

21. Tufvesson, P.; Lima-Ramos, J.; Jensen, J.S.; Al-Haque, N.; Neto, W.; Woodley, J.M. Process considerations for the asymmetric synthesis of chiral amines using transaminases. Biotechnol. Bioeng. 2011, 108, 1479-1493. [CrossRef]

22. Otzen, M.; Palacio, C.; Janssen, D.B. Characterization of the caprolactam degradation pathway in Pseudomonas jessenii using mass spectrometry-based proteomics. Appl. Microbiol. Biotechnol. 2018, 102, 6699-6711. [CrossRef] [PubMed]

23. Palacio, C.M.; Rozeboom, H.J.; Lanfranchi, E.; Meng, Q.; Otzen, M.; Janssen, D.B. Biochemical properties of a Pseudomonas aminotransferase involved in caprolactam metabolism. FEBS J. 2019, 286, 4086-4102. [CrossRef] [PubMed]

24. Meng, Q.; Capra, N.; Palacio, C.M.; Lanfranchi, E.; Otzen, M.; van Schie, L.Z.; Rozeboom, H.J.; Thunnissen, A.-M.W.H.; Wijma, H.J.; Janssen, D.B. Robust $\omega$-Transaminases by Computational Stabilization of the Subunit Interface. ACS Catal. 2020, 10, 2915-2928. [CrossRef]

25. Calvelage, S.; Dörr, M.; Höhne, M.; Bornscheuer, U.T. A Systematic Analysis of the Substrate Scope of (S)and (R)-Selective Amine Transaminases. Adv. Synth. Catal. 2017, 359, 4235-4243. [CrossRef]

26. Huisman, G.W.; Collier, S.J. On the development of new biocatalytic processes for practical pharmaceutical synthesis. Curr. Opin. Chem. Biol. 2013, 17, 284-292. [CrossRef] [PubMed]

27. Cho, B.-K.; Park, H.-Y.; Seo, J.-H.; Kim, J.; Kang, T.-J.; Lee, B.-S.; Kim, B.-G. Redesigning the substrate specificity of $\omega$-aminotransferase for the kinetic resolution of aliphatic chiral amines. Biotechnol. Bioeng. 2008, 99, 275-284. [CrossRef] [PubMed]

28. Nobili, A.; Steffen-Munsberg, F.; Kohls, H.; Trentin, I.; Schulzke, C.; Höhne, M.; Bornscheuer, U.T. Engineering the Active Site of the Amine Transaminase from Vibrio fluvialis for the Asymmetric Synthesis of Aryl-Alkyl Amines and Amino Alcohols. ChemCatChem 2015, 7, 757-760. [CrossRef]

29. Genz, M.; Melse, O.; Schmidt, S.; Vickers, C.; Dörr, M.; van den Bergh, T.; Joosten, H.-J.; Bornscheuer, U.T. Engineering the Amine Transaminase from Vibrio fluvialis towards Branched-Chain Substrates. ChemCatChem 2016, 8, 3199-3202. [CrossRef]

30. Midelfort, K.S.; Kumar, R.; Han, S.; Karmilowicz, M.J.; McConnell, K.; Gehlhaar, D.K.; Mistry, A.; Chang, J.S.; Anderson, M.; Villalobos, A.; et al. Redesigning and characterizing the substrate specificity and activity of Vibrio fluvialis aminotransferase for the synthesis of imagabalin. Protein Eng. Des. Sel. 2013, 26, 25-33. [CrossRef]

31. Ericsson, U.B.; Hallberg, B.M.; DeTitta, G.T.; Dekker, N.; Nordlund, P. Thermofluor-based high-throughput stability optimization of proteins for structural studies. Anal. Biochem. 2006, 357, 289-298. [CrossRef] [PubMed]

32. Boivin, S.; Kozak, S.; Meijers, R. Optimization of protein purification and characterization using Thermofluor screens. Protein Expr. Purif. 2013, 91, 192-206. [CrossRef] [PubMed]

33. Krieger, E.; Vriend, G. YASARA View-Molecular Graphics for 1139 all Devices_from Smartphones to Workstations. Bioinformatics 2014, 30, 2981-2982. [CrossRef] [PubMed] 
34. Conway, P.; Tyka, M.D.; DiMaio, F.; Konerding, D.E.; Baker, D. Relaxation of backbone bond geometry improves protein energy landscape modeling. Protein Sci. 2014, 23, 47-55. [CrossRef] [PubMed]

35. Richter, F.; Leaver-Fay, A.; Khare, S.D.; Bjelic, S.; Baker, D. De Novo Enzyme Design using Rosetta3. PLoS ONE 2011, 6, e19230. [CrossRef]

Publisher's Note: MDPI stays neutral with regard to jurisdictional claims in published maps and institutional affiliations.

(C) 2020 by the authors. Licensee MDPI, Basel, Switzerland. This article is an open access article distributed under the terms and conditions of the Creative Commons Attribution (CC BY) license (http://creativecommons.org/licenses/by/4.0/). 